\section{ARTíCULO CORTO DE INVESTIGACIÓN CIENTÍFICA}

DOI: 10.47864/SE(50)2020p31-39_123
Sociedad Colombiana de la Ciencia del Suelo

\title{
DINÁMICA DE LA DESCOMPOSICIÓN DE RESIDUOS ORGÁNICOS
}

\author{
Carmen Rosa Bonilla Correa $\rrbracket^{l}$, Juan Díaz ${ }^{2}$, Carla Gil ${ }^{2}$, Karla Girón ${ }^{2}$, Mónica León ${ }^{2}$, Otoniel Ortiz ${ }^{2}$, \\ Angie Suarez ${ }^{2}$
}

1 Ingeniera Agrónoma, Maestría en Suelos y Aguas. Universidad Nacional de Colombia

\section{$\triangle$ Je-mail:}

crbonillac@unal.edu.co

2 Estudiantes maestría en Ciencias Agrarias, Universidad Nacional de Colombia, sede Bogotá

Palabras claves:

Litter

bags,mineralización, materia orgánica del suelo.

\section{RESUMEN}

Teniendo en cuenta que el material orgánico del suelo es un componente de importancia en la estructura y funcionamiento de los ecosistemas, principalmente en los cambios geoquímicos y en la fertilidad del suelo y que la dinámica de su mineralización depende principalmente de la actividad de los microorganismos, del tipo de moléculas orgánicas que predomine en los materiales que llegan al suelo y de las condiciones ambientales como temperatura y precipitación, se realizó el siguiente trabajo con el objetivo de evaluar los cambios en los materiales orgánicos que llegan al suelo dependiendo de ubicación en el perfil del suelo. Se utilizó la metodología modificada de Litter Bags colocando $300 \mathrm{~g}$ de material de origen vegetal, principalmente gramíneas, en bolsas plásticas con agujeros de $1 \mathrm{~mm}$ aproximadamente. Se utilizaron bolsas blancas para simular la descomposición del material en superficie (colocadas sobre la superficie) y negras (enterradas $20 \mathrm{~cm}$ ) para simular la descomposición dentro del perfil del suelo. El número total de bolsas para cada caso fue de 12, de tal manera que se realizó un muestreo de tres bolsas por cada condición a los 22, 35, 56 y 78 días después del establecimiento y al inicio del ensayo. En la muestra inicial de determinó la composición del tejido vegetal, la materia seca y el contenido de humedad. En los muestreos intermedios se midió la materia seca y la humedad y en el muestreo final, además de lo anterior se volvió a determinar la composición química del tejido vegetal. Se evidenció que la pérdida de materia seca es un indicador de la velocidad de descomposición del material ya que el material orgánico enterrado al conservar la humedad, favoreció el proceso de pérdida de materia seca y por lo tanto una mayor tasa de mineralización. El contenido de N pasó de $1.11 \%$ a $2.45 \%$ en el material enterrado y de $1.11 \%$ a $1.96 \%$ en el material depositado sobre la superficie, lo cual indica la conveniencia de enterrar los materiales orgánicos.

\section{DYNAMICS OF ORGANIC WASTE DECOMPOSITION}

Keywords:

Litter bags,mineralization ,soil organic matter

\begin{abstract}
Taking into account that the organic material of the soil is an important component in the structure and functioning of ecosystems, mainly in geochemical changes and soil fertility, and that the dynamics of its mineralization depends mainly on the activity of microorganisms, the type of organic molecules that predominate in the materials that reach the soil and the environmental conditions such as temperature and precipitation, the following work was carried out with the aim of evaluating the changes in the organic materials that reach the soil depending on location in the profile soil. The modified Litter Bags methodology was used, placing $300 \mathrm{~g}$ of plant waste material, mainly grasses, in plastic bags with holes of approximately $1 \mathrm{~mm}$. White bags were used to simulate the decomposition of the material on the soil surface (placed on the surface) and black (buried $20 \mathrm{~cm}$ ) to simulate the decomposition within the soil profile. The total number of bags for each case was 12, in such a way that a sampling of three bags was performed for each condition at the beginning of the trial and at 22, 35, 56 and 78 days after establishment. In the initial sample, the composition of the plant tissue, the dry matter and the moisture content were determined. In the intermediate samplings, the dry matter and humidity were measured and in the final sampling, in addition to the above, the chemical composition of the plant tissue was determined again. It was evidenced concluded that the loss of dry matter which is an indicator of the decomposition rate of the material, was larger in the buried organic material than in that placed on the soil surface due to the moisture content which favored the process of mineralization on the buried one.
\end{abstract}




\section{INTRODUCCIÓN}

La materia orgánica del Suelo (MOS) contiene la mayor cantidad de $\mathrm{C}$ de la superficie de la tierra $\left(2,157-2,293 \mathrm{Pg} ; \mathrm{Pg}=10^{15} \mathrm{~g}\right)$, el doble del presente en la atmósfera (760 Pg), y de 2 a 3 veces mayor que el de todos los organismos vivientes en el conjunto de ecosistemas terrestres (Batjes, 1996). Además, debido a su presencia y su participación en casi todos los procesos del suelo constituye un factor determinante de la calidad y de la salud de los suelos, la cual se refiere a las características biológicas, físicas y químicas que son esenciales para una productividad sostenible a largo plazo con el mínimo de impacto ambiental (Arias et al., 2005).

Una característica de la MOS que ha adquirido especial relevancia ambiental en los últimos años es su elevado potencial para secuestrar $\mathrm{C}$ de forma estable, principalmente a través de los procesos de humificación y de formación de complejos organominerales, que conducen o favorecen la formación de formas estables y refractarias de $\mathrm{C}$ orgánico. Las investigaciones sobre secuestro de carbono en el suelo, pretenden establecer los factores responsables del balance humificación/mineralización, que permitirían diferenciar distintos tipos de suelo en función de su comportamiento como fuente o como sumidero de carbono, y evaluar su trascendencia sobre aspectos atmosféricos que inciden en el cambio climático global. Para almacenar $\mathrm{C}$ y así disminuir la concentración de $\mathrm{CO}_{2}$ emitido por la actividad humana será necesario intervenir sobre los sumideros de $\mathrm{C}$ situados en la biosfera continental (Arias et al., 2005).

La descomposición del litter en los ecosistemas terrestres juega un papel importante en el ciclo global del carbono a través de las emisiones de $\mathrm{CO}_{2}$ a la atmósfera. El cambio climático global puede afectar la descomposición del litter y, en consecuencia, alterar el flujo de $\mathrm{CO}_{2}$ del suelo, tanto física como microbiológicamente (Chapin III et al., 2011). Por lo tanto, los aspectos de la descomposición de la hojarasca son cruciales para la predicción del flujo de $\mathrm{CO}_{2}$ del suelo en condiciones de cambio climático global.

La descomposición del litter u hojarasca se ve afectada tanto por factores bióticos y abióticos (Cotrufo et al., 2009). Un factor biótico importante son los microorganismos descomponedores que transforman el litter a través de catabolismo (Lifei et al., 2017).

La descomposición es un proceso complejo regulado por las interacciones entre los organismos (fauna y microrganismos), factores físicos medioambientales (principalmente temperatura y humedad) y la calidad del recurso orgánico (concentración de lignina, nitrógeno y polifenoles condensados y solubles). A medida que avanza la descomposición, los materiales solubles y particulados del litter, los tejidos de los organismos y los productos del metabolismo microbiano se separan de la fuente original mediante actividades de lixiviación, fragmentación física y alimentación animal. Posteriormente esos productos son transportados por el viento, el agua y la gravedad a microhábitats en el suelo que tienen condiciones diferentes que regulan la descomposición (Anderson e Ingram, 1993).

Cuando el sustrato en el suelo está disponible, la actividad microbiana del suelo aumenta exponencialmente con la temperatura del suelo, y la actividad microbiana a menudo se duplica con un aumento de temperatura de $10{ }^{\circ} \quad \mathrm{C}$ (Kirschbaum, 1995). Los microorganismos también pueden ser limitados por la humedad del suelo. A medida que aumentan la temperatura, la humedad del suelo asume un papel cada vez más importante para mantener altas tasas de actividad microbiana (Peterjohn et al., 1994). Como resultado, las tasas de descomposición del litter 
fresco aumentan con el aumento tanto de la temperatura como de la humedad (precipitación) (Meentemeyer 1978).

Este patrón general de descomposición también puede verse influenciado por la variabilidad en la calidad del litter. La calidad se refiere a las características químicas, físicas) que influyen en la susceptibilidad del litter a la descomposición. El litter que contiene altas concentraciones de compuestos lábiles (azúcares, aminoácidos) tiende a descomponerse rápidamente porque estos compuestos pueden metabolizarse fácilmente por microorganismos del suelo o ser lixiviados. Compuestos estructurales lábiles tales como la celulosa es rápidamente transformada por exoenzimas en subunidades de azúcar, que nuevamente son metabolizados fácilmente por organismos microbianos. Por el contrario, moléculas recalcitrantes como la lignina y la quitina son demasiado grandes para pasar a través de las membranas celulares, y en cambio son procesadas lentamente por enzimas extracelulares. La estructura química irregular y enlaces complejos hacen que estos compuestos sean difíciles de metabolizar y a largo plazo proporcionan una liberación lenta de $\mathrm{N}$ y $\mathrm{P}$ para el crecimiento microbiano.

La descomposición del litter y la liberación de $\mathrm{N}$ está positivamente relacionada con la calidad inicial del litter. En las primeras etapas de descomposición, la proporción de C: $\mathrm{N}$ puede ser el mejor predictor de pérdida de masa $\mathrm{y}$ liberación de $\mathrm{N}$, con un incremento positivo de la lignina en las etapas posteriores de la descomposición del litter.

Las diferencias en la calidad inicial del litter (como la relación lignina: $\mathrm{N}$ y lignina: celulosa) alteran la descomposición del litter y las tasas de liberación en las etapas tempranas de descomposición del litter. A medida que la calidad del sustrato disminuye durante la descomposición, la calidad inicial litter tiene una influencia decreciente en la descomposición en la etapa tardía. En esta etapa, las tasas de descomposición son más influenciadas por el clima, la textura del suelo, y las fuentes exógenas de $\mathrm{C}$ lábil y nutrientes.

La descomposición del litter y las tasas de liberación de $\mathrm{N}$ están negativamente relacionadas con las estimaciones iniciales de $\mathrm{N}$ y a la calidad del litter. Un alto contenido de $\mathrm{N}$ puede retrasar las tasas de descomposición del litter, particularmente si los niveles de lignina también son altos.

El método de Litterbags (LB) es el más usado para determinar las tasas de descomposición del litter. El LB determina la tasa de descomposición de la hojarasca o litter evaluando la pérdida de masa o elementos del litter en bolsas de malla colocadas en la superficie del suelo o en la capa superior del suelo durante un período de tiempo determinado. Es un método simple y económico (Kampichler y Bruckner, 2009). Sin embargo, el tamaño de la malla de la bolsa puede afectar la descomposición de la hojarasca a través de los cambios en la fauna del suelo que puede acceder y romper y digerir la hojarasca en las bolsas y eventualmente estimular la actividad microbiana en la bolsa (Bokhorst y Wardle, 2013).

Sin embargo, este método ha mostrado algunas limitaciones ya que la duración del experimento debe ser decidido a priori basado en una supuesta tasa de descomposición. El tamaño de la malla, incluso grande, puede excluir parte de la macrofauna $\mathrm{o}$ puede inducir la pérdida de material, y la hojarasca confinada puede modificar las condiciones microclimáticas (Hutchinson et al., 1990; Virzo De Santo et al., 1993), citados por Kurz-Besson et al, 2005.

Teniendo en cuenta la importancia que radica la transformación de los residuos orgánicos en el suelo, el desarrollo de la presente investigación tuvo como objetivo valorar la tasa de descomposición de residuos orgánicos a partir de 
la pérdida de peso de residuos orgánicos colocado sobre y bajo la superficie del suelo.

\section{MATERIALES Y MÉTODOS}

El ensayo se realizó en la zona de invernaderos de la Facultad de Ciencias Agrarias de Universidad Nacional de Colombia, sede Bogotá. Se seleccionó una mezcla de residuos orgánicos (pastos, hojas, ramas), se homogenizó y se colocaron $300 \mathrm{~g}$ de la muestra en bolsas plásticas agujereadas uniformemente con un diámetro aproximadamente de 1 a $2 \mathrm{~mm}$. Del material homogenizado se tomó una muestra a la cual se le determinó humedad, materia seca y análisis de tejido vegetal.

Se utilizaron bolsas blancas para simular la descomposición sobre la superficie y negras para simular la descomposición bajo el perfil. Las bolsas blancas se colocaron sobre la superficie del suelo. Para las bolsas negras se cavó una zanja de $25 \mathrm{~cm}$ y a lo largo de ella se ubicaron las bolsas las cuales se cubrieron con suelo y con la capa vegetal que se encontraba sobre la zanja.

El número total de bolsas para cada tratamiento (sobre y bajo el perfil del suelo) fue de 12. Cada tres semanas se realizó un muestreo consistente en recoger las primeras tres bolsas de cada tratamiento. Las tres bolsas corresponden a tres repeticiones de cada uno de los tratamientos. El material de las bolsas se pesó nuevamente y se le determinó el contenido de humedad y el porcentaje de materia seca. En el último muestreo también se realizó un análisis de tejido vegetal con el fin de compararlo con el análisis inicial.

\section{RESULTADOS Y DISCUSIÓN}

En la Figura 1 se observa que el material enterrado en bolsas negras presentó a lo largo del tiempo un incremento en el contenido de humedad. La humedad inicial correspondió a $50 \%$ y en el muestreo final fue de $69 \%$. Este contenido de agua favorece de manera directa la actividad de la fauna y de los microorganismos del suelo, los cuales utilizan los residuos orgánicos como fuente de energía haciendo que los residuos se transformen a través de procesos de oxidación mediados por enzimas. La actividad biológica igualmente se ve favorecida por la temperatura, que al interior del perfil se conserva y facilita el metabolismo de los microorganismos. Por el contrario, los residuos ubicados sobre la superficie del suelo, al estar expuestos directamente a la acción de la temperatura, conservan menos humedad y por lo tanto la actividad de los organismos es menor en comparación con la actividad biológica que se realiza al interior del perfil del suelo (Figura 1).

Según Céspedes (2007) el clima es el principal factor exógeno que actúa sobre la materia orgánica a través de la influencia que ejerce la temperatura y humedad sobre la producción de biomasa, aporte y acumulación de residuos orgánicos, actividad biológica y tasa de biodegradación.

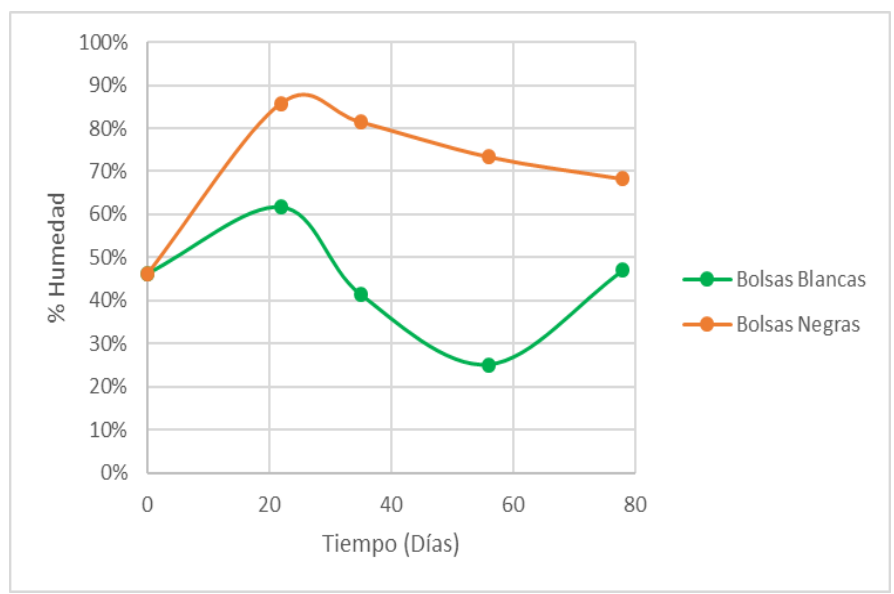

Figura 1. Contenido de humedad a través del tiempo de residuos orgánicos expuestos sobre la superficie del suelo (bolsas blancas) y al interior del perfil (bolsas negras).

La pérdida de materia seca es un indicador de la velocidad de descomposición del material orgánico adicionado. En la Figura 2 se evidencia que el material orgánico enterrado (bolsas negras) perdió mayor porcentaje de masa seca en 
comparación al a los residuos expuestos sobre la superficie del suelo (bolsas blancas). Esta condición está directamente relacionada con una mayor actividad biológica, la cual se vio favorecida por el contenido de humedad y la disponibilidad de oxígeno que hace posible la respiración de los organismos y la oxidación de las moléculas orgánicas que constituyen los residuos orgánicos.

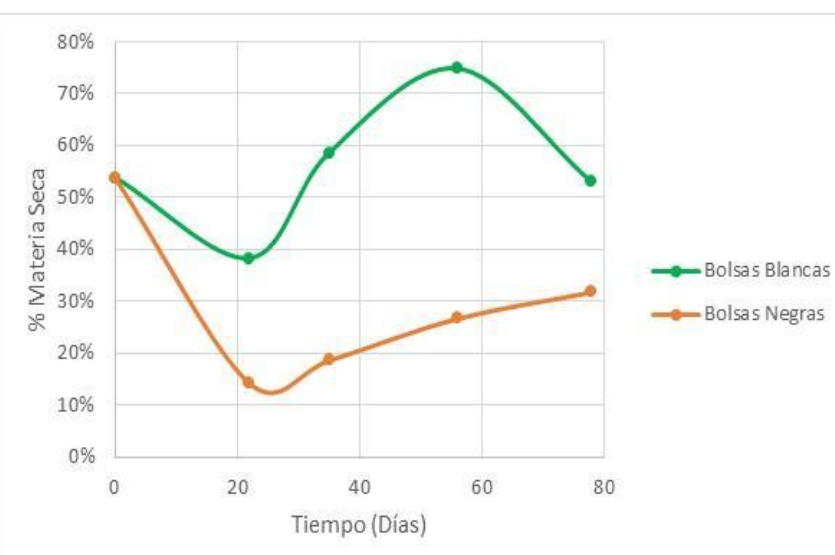

Figura 2. Contenido de materia seca a través del tiempo de residuos orgánicos expuestos sobre la superficie del suelo (bolsas blancas) y al interior del perfil (bolsas negras).

La temperatura es una de las condiciones ambientales más importante que determinan la velocidad de descomposición de los residuos orgánicos. Un cambio en la temperatura tiene una influencia directa sobre cada uno de los organismos dentro de la comunidad. La humedad debe ser adecuada para que la descomposición proceda, los microorganismos crecen fácilmente en medios líquidos donde el suministro de oxígeno es adecuado. Estos factores incidieron directamente en los resultados observados en la Figura 2 ya que el material que se expuso en bolsas negras se conservó más húmedo permitiendo así mayor acción de los microorganismos mientras que el de las bolsas blancas permaneció con porcentajes de humedad menores al $50 \%$ lo que reduce la actividad de estos.
Otro factor importante es la profundidad a la que se expusieron los residuos, los residuos enterrados en los primeros centímetros del perfil del suelo estuvieron expuestos a una mayor cantidad de organismos, en comparación al material colocado sobre la superficie del suelo, donde el proceso de descomposición es afectado por la disponibilidad de agua.

La calidad de los residuos es otro factor que afecta directamente la velocidad de descomposición. Materiales orgánicos con baja relación $\mathrm{C} / \mathrm{N}(>12)$ y con alto contenido de moléculas orgánicas lábiles como azúcares, aminoácidos, tienden a descomponerse rápidamente debido a que esos compuestos son rápidamente metabolizados por los organismos del suelo o se pierden a través del perfil por lixiviación. Por el contrario, compuestos con moléculas altamente recalcitrantes como lignina y quitina con alta relación $\mathrm{C} / \mathrm{N}(>30)$, son lentamente mineralizables, lo cual indirectamente contribuye al mejoramiento de algunas propiedades físicas del suelo como la aireación y la infiltración del agua, además de que constituyen materiales orgánicos fuente de alimento para organismos especializados que actúan sobre esos materiales liberando nuevos compuestos que contribuyen a los procesos de humificación (Giardina et al. 2001).

A medida que los organismos del suelo descomponen los residuos orgánicos, los nutrientes en exceso (nitrógeno, fósforo y azufre) son liberados dentro del suelo en formas que pueden ser usadas por las plantas (disponibilidad de nutrientes). Los productos de deshecho producidos por los microorganismos contribuyen a la formación de la materia orgánica del suelo. Los materiales de desecho son más difíciles de descomponer que el material original de las plantas y los animales, pero pueden ser usados a su vez por un gran número de organismos.

La descomposición de la materia orgánica y la liberación del carbono son procesos aeróbicos, lo 
que significa que los microorganismos necesitan oxígeno y, por lo tanto, los residuos sobre la superficie del suelo generan un ciclo del carbono más lento debido a que están expuestos a menos microorganismos y entonces estos decaen más lentamente dando lugar a la producción de humus que es más estable y libera menos dióxido de carbono a la atmósfera; cuando se ara los residuos son incorporados en el suelo junto con el aire y se ponen en contacto con muchos microorganismos lo cual acelera el ciclo del carbono. La descomposición es más rápida, lo que trae como resultado menos formación de humus estable y liberación del dióxido de carbono a la atmósfera y, por lo tanto, una reducción de la materia orgánica.

En la figura 3 se observa que la ganancia en peso fresco de los residuos orgánicos de las bolsas negras es por contenido de agua producto principalmente de la precipitación mientras que la pérdida de peso fresco del material de las bolsas blancas es efecto principal de la evaporación del agua por su exposición directa a la radiación.

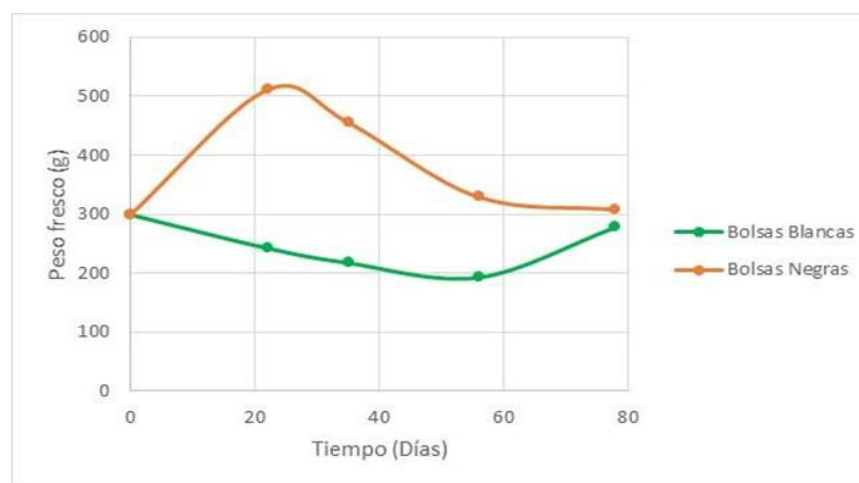

Figura 3. Evolución del peso fresco de residuos orgánicos expuestos sobre la superficie del suelo (bolsas blancas) y al interior del perfil (bolsas negras)

En la tabla 1 se presentan los resultados de análisis de tejido vegetal realizados al inicio del experimento y al final tanto para el material orgánico de las bolsas blancas como el de las bolsas negras. En el material orgánico de las bolsas blancas se observa un aumento en la concentración de la mayoría de los elementos con excepción del $\mathrm{Ca}$ y el $\mathrm{Mg}$. Para el caso de los residuos orgánicos de las bolsas negras se dio un aumento en la concentración de la mayoría de los elementos con excepción del K y el S.

En las primeras etapas de degradación de la materia orgánica se producen intensas pérdidas de carbono mientras que el nitrógeno es incorporado a la fauna microbiana, lo que conduce a que la relación $\mathrm{C} / \mathrm{N}$ disminuya. Con relación al nitrógeno, el material de las bolsas negras acumuló un $25 \%$ más de $\mathrm{N}$ en comparación al de las bolsas blancas, lo que permite inducir que aun sin tener un valor de carbono estimado para las muestras, que los residuos orgánicos de las bolsas negras liberaron una mayor cantidad de $\mathrm{N}$ como consecuencia de su actividad. El papel de los microorganismos es decisivo para el desarrollo de este proceso. Los microorganismos necesitan del carbono como fuente de energía (oxidan el $\mathrm{C}$ y lo devuelven a la atmósfera como $\mathrm{CO}_{2}$ ) y también del nitrógeno para incorporarlo a su protoplasma.

Analizando la concentración de fósforo durante la descomposición de los residuos orgánicos se puede observar que ésta aumentó en un 38,23\% y un $47,05 \%$ para el material de las bolsas blancas y negras, respectivamente, en comparación a la concentración inicial (Tabla 1). Este comportamiento fue reportado para muchos ecosistemas forestales, encontrándose ciclos de inmovilización y liberación para elementos, especialmente fósforo (Wesemael, 1993), quien halló un incremento de las concentraciones de fósforo durante la descomposición en todos los 
tipos de hojarascas analizadas. Prause (1997) encontró diferentes ritmos de liberación de $\mathrm{P}$ en un estudio realizado con especies nativas del Parque Chaqueño, que fueron atribuidas a la inmovilización microbiana (Swift et al. 1979) y a la contaminación del material vegetal confinado en las bolsas, con el suelo mineral donde se hallan fijadas (Wesemael, 1993). El fósforo es el elemento que queda más retenido en el material vegetal en comparación a elementos como el nitrógeno y el potasio.

$\mathrm{Si}$ realizamos el cálculo del porcentaje de nutriente remanente (teniendo en cuenta que se usó la misma masa de residuos orgánicos para los análisis) expresado en la siguiente fórmula:
$\% \mathrm{NR}=$ _. Concentración final $\times$ Masa 100

\section{Concentración final x Masax}

Para el caso del potasio estimado para los residuos orgánicos de las bolsas negras este corresponde a un $56,54 \%$, lo que representa una rápida pérdida atribuida a que este elemento no se encuentra formando parte de ninguna estructura en el vegetal y siempre se lo halla como ion libre asociado a sales muy solubles, el cual puede ser removido por efecto de la precipitación (De La Horra, et al. 2000). Adicionalmente, se evidencia que las condiciones a las que se expusieron las bolsas negras favorecieron esta liberación en comparación a las bolsas blancas donde incluso la concentración de potasio aumento.

Tabla 1. Análisis de tejido vegetal de residuos orgánicos

\begin{tabular}{|l|l|l|l|l|l|l|l|l|l|l|l|}
\hline & $\mathrm{N}$ & $\mathrm{P}$ & $\mathrm{Ca}$ & $\mathrm{K}$ & $\mathrm{Mg}$ & $\mathrm{Cu}$ & $\mathrm{Fe}$ & $\mathrm{Mn}$ & $\mathrm{Zn}$ & $\mathrm{B}$ & \multicolumn{7}{|c|}{$\mathrm{mg} / \mathrm{kg}$} \\
\hline & \multicolumn{9}{|c|}{$\%$} & \multicolumn{7}{|c|}{} \\
\hline Inicial & 1,11 & 0,34 & 1,16 & 2,37 & 0,22 & 9,16 & 432 & 76,1 & 69,4 & 11,4 & 2946 \\
\hline Análisis Final BB* & 1,96 & 0,47 & 0,62 & 4,65 & 0,22 & 12,1 & 765 & 79,9 & 92,4 & 25,8 & 3325 \\
\hline $\begin{array}{l}\text { Análisis Final } \\
\text { BN** }\end{array}$ & 2,45 & 0,5 & 2,06 & 1,34 & 0,33 & 13 & 1706 & 146 & 137 & 34,1 & 2784 \\
\hline
\end{tabular}

*Bolsa blanca

**Bolsa negra

\section{CONCLUSIONES}

La calidad de los residuos orgánicos, las condiciones de temperatura y humedad y la ubicación de los residuos en el perfil, afectan directamente la actividad de los microrganismos y por lo tanto el proceso de mineralización.

\section{REFERENCIAS}

Anderson, J.A. and Ingram, J.S.I (eds). 1993. Tropical Soil Biology and Fertility: A Handbook of Methods. Second edition. CAB International. $221 \mathrm{p}$.

Arias, M.E., González-Pérez, J.A., GonzálezVila, F.J., Ball, A.S., 2005. Soil health-a new 
challenge for microbiologists and chemists. International Microbiology. 8, 1-9.

Asociación Vida Sana. (2010). Microorganismos del suelo y biofertilización. Recuperado http://ec.europa.eu/environment/life/project/Proje cts/index.cfm?fuseaction=home.showFile\&rep=file \&fil=CROPS-FOR-BETTER-SOIL_formacion-5.pdf.

Batjes, N.H., 1996. Total carbon and nitrogen in the soils of the world. Eur J Soil Sci. 47: 63-151

Bonilla, Gómez y Sánchez. (2002). Cuaderno ambiental del suelo: los organismos que lo habitan (5). Recuperado de http://www.uneditorial.net/uflip/El-suelo-losorganismos-que-lo-habitan/pubData/source/Elsuelo-los-organismos-que-lo-habitan_Uflip.pdf.

Bokhorst S, Wardle D.A. 2013. Microclimate within litter bags of different mesh size: Implications for the 'arthropod effect' on litter decomposition. Soil Biology and Biochemistry $58,147-152$.

Céspedes, C. (2007). Dinámica de la materia orgánica y de algunos parámetros fisicoquímicos en Molisoles, en la conversión de una pradera a cultivo forestal en la región de Piedras Coloradas - Algorta (Uruguay) (Tesis de doctorado). Instituto Nacional Politécnico de Toulouse, Toulouse, Francia.

Chapin III FS, Matson PA, Vitousek P, 2011: Principles of terrestrial ecosystem ecology. Second edition. Springer, New York, USA. pp.529.

Cotrufo MF, Del Galdo I, Piermatteo D, 2009: Litter decomposition: concepts, methods and future perspectives. In: Soil carbon dynamics: an integrated methodology (ed. by Kutsch, W. L. et al.). Cambridge University Press, Cambridge, pp 76-90

De La Horra, A.M.; D. Effron; R.M. Palma; y J. Prause. 2000. Liberación y dinámica de calcio, potasio, magnesio y sodio proveniente de la descomposición de hojarasca en un bosque subtropical argentino.Agrochímica. VXLIV (34):107-114.

Digon, A. (Productor). (2013). Regenerando la tierra, alma y sociedad [YouTube]. De https://www.youtube.com/watch?v=ngsHcF68Cml.

FAO. (2015). Suelos y biodiversidad: los suelos albergan una cuarta parte de la biodiversidad de nuestro planeta. Recuperado de http://www.fao.org/3/a-i4551s.pdf.

Giardina CP, Ryan MG, Hubbard RM, Binkley D. 2001. Tree species and soil textural controls on carbon and nitrogen mineralization rates. Soil Science Society of America Journal 65: 1272-1279

Howard, S. (1940). Un Testamento Agrícola. Recuperado de file://C:/Users/uniminuto/Downloads/Un\%20T estamento\%20Agr\%C3\%ADcola_ Howzard\%20(1).pdf.

Kampichler C, Bruckner A. 2009.: The role of microarthropods in terrestrial decomposition: a meta-analysis of 40 years of litterbag studies. Biological Reviews 84, 375-389

Kirschbaum MUF (1995). The temperature dependence of soil organic matter decomposition, and the effect of global warming on soil organic $\mathrm{C}$ storage. Soil Biology and Biochemistry 27: 753-760.

Kurz-Besson; K., Couteauxa, M.M., Thiery, J.M., Bergc, B. and Remacle, J. 2005. A comparison of litterbag and direct observation methods of Scots pine needle decomposition measurement. Soil Biology \& Biochemistry 37, 2315-2318.

Lifei, S., Munemasa, T., Naishin,L., Tomotsugu, $\mathrm{Y}$. and Takaschi, $\mathrm{H}$. 2017.Comparison of litter-bag and chamber methods for measuring $\mathrm{CO} 2$ emissions from 
leaf litter decomposition in a temperate forest. Journal of Agricultural Meteorology 73(2) 5967.

Meentemeyer V (1978). Macroclimate and lignin control of litter decomposition rates. Ecology 59: 465472

Pascual, R y Vanegas, S. (2011). La materia orgánica del suelo - papel de los microorganismos. Recuperado de https://www.ugr.es/ cjl/MO\%20en\%20suelos.pdf.

Peterjohn WT, Melillo JM, Steudler PA, Newhrk KM, Bowles FP, Aber JD (1994) Responses of trace gas fluxes and $\mathrm{N}$ availability to experimentally elevated soil temperatures. Ecological Applications 4: 617-625

Prause, J. 1997. Aporte de las principales especies forestales a la dinámica de la materia orgánica y de los nutrientes en un monte nativo del Parque Chaqueño húmedo. M.Sc. Tesis. Facultad de Agronomía.Universidad de Buenos Aires. Argentina. 205 p.

Wesemael, B. 1993. Litter decomposition and nutrient distribution in humus profiles in some mediterranean forests in southern Tuscany. Forest Ecology and Management. 57: 99-114. 